\section{Etiquetado de alimentos en Ecuador: Respuesta a la carta de Fornasini et al.}

Estimado Editor:

En respuesta a la carta de Fornasini et al. ${ }^{a}$ destacamos que la metodología propuesta en nuestro artículo -basada en la triangulación de información proveniente de entrevistas en profundidad a informantes claves, revisión bibliográfica y estudios de mercado de Euromonitor y Nielsen- es la más apropiada para responder a preguntas de investigación como las planteadas, dado que permite reducir o compensar las deficiencias de una única estrategia, incrementa la validez de los resultados y mejora la profundidad y la consistencia de los procedimientos metodológicos (1).

En la carta de Fornasini et al. se confunde la definición de datos primarios y secundarios; la información proveniente de entrevistas en profundidad representa datos primarios y no secundarios como apuntan. Por otro lado, se afirma equivocadamente que el uso de datos secundarios es una limitación metodológica; sin embargo, las bases de datos econométricos, como Euromonitor y Nielsen, son perfectamente válidas para responder al objetivo de nuestro estudio, tal como lo prueba la amplia utilización y reconocimiento de estas fuentes en estudios de mercados de productos de consumo publicados en revistas internacionales de gran prestigio, credibilidad y rigor científico $(2,3)$.

Respecto de la afirmación de que se debería "haber investigado a profundidad el nivel de entendimiento y el efecto en la compra de los consumidores..." nuestro artículo aclara que dicha pregunta fue respondida en estudios realizados en la etapa de previa a la adopción del etiquetado tipo semáforo (ETS) y que demostraron la adecuada comprensión de esta opción. Esta observación fue posteriormente ratificada en el estudio de Freire et al. (4).

Fornasini et al. afirman que -según estudios realizados por su grupo- "existe una discordancia" entre el rotulado tradicional y el ETS; sin embargo, no se dan detalles de la investigación ni se cita dónde fueron publicados estos resultados, por lo cual consideramos que se trata de una opinión o especulación, sobre la cual no podemos emitir comentario técnico.

Sobre la supuesta inconsistencia entre la política del etiquetado y la tendencia en el consumo de bebidas azucaradas, ratificamos lo expresado en el artículo respecto que no se puede esperar que una sola medida -en solo dos años de implementación- revierta la tendencia de décadas en el consumo de productos ultraprocesados. Por otro lado, una lectura detallada del artículo permite comprender que, si bien se mantuvo el

\footnotetext{
https://doi.org/10.26633/RPSP.2018.37
}

incremento del consumo de productos ultraprocesados, la tendencia de crecimiento ha disminuido.

Sobre la sugerencia de "considerar otras posibilidades de rotulado alimentario que sea más comprensivo en la evaluación de la calidad nutricional de los alimentos procesados" es importante destacar que ello implicaría un estudio de comprensión por parte de la población como el realizado para el ETS. Por otro lado, el tipo de propuesta sugerida fue diseñada para contextos muy distintos del ecuatoriano y contradice la evidencia que demuestra que cuanto más simple y directa es la información del etiquetado, como en los casos de Ecuador y Chile, mejor es la compresión y la toma de decisión de compras por parte de la población (5).

Llama la atención que Fornasini et al. ponen en duda la estrecha asociación entre el incremento del consumo de productos alimenticios ultraprocesados ricos en sodio, azúcar y/o grasas, con la epidemia de enfermedades no transmisibles (ENT). El informe de la Organización Mundial de la Salud sobre este tema indica que las dietas malsanas son uno de los 4 factores de riesgo que más muertes causan y quitan años de vida saludable a la población (6). Los dos artículos citados -financiados por empresas que fabrican productos con altas concentraciones de estos nutrientes o medicamentos para el tratamiento de las ENThan presentado conclusiones erróneas (7) y fueron mal interpretados, pues muestran la relación entre el consumo excesivo de estos nutrientes y las ENT.

Finalmente, ratificando lo planteado en las conclusiones del trabajo -respecto a que las medidas regulatorias confrontan con una férrea oposición por parte de la industria de productos ultraprocesados- consideramos importante transparentar el hecho que autores de la carta tienen estrechos vínculos con este sector, ya sea por haber recibido financiamiento para alguna de sus investigaciones o por integrar directorios de instituciones con fuerte presencia de la industria $(8,9,10)$, lo que representa un conflicto de interés como ha sido demostrado en otros casos (11).

\section{Conflictos de interés. Ninguno declarado.}

Declaración. Las opiniones expresadas en este manuscrito son responsabilidad del autor y no reflejan necesariamente los criterios ni la política de la RPSP/PAJPH y/o de la OPS.

\section{Adrián Díaz Organización Panamericana de la Salud, Quito, Ecuador}

Forma de citar Díaz A. Etiquetado de alimentos en Ecuador: Respuesta a Fornasini et al. [carta]. Rev Panam Salud Publica. 2018;42:e70. https:/ /doi.org/10.26633/RPSP.2018.70 


\section{REFERENCIAS}

1. Thurmond VA. The Point of Triangulation. J Nurs Scholarsh. 2001; 33:253-8.

2. Moodie R, Stuckler D, Monteiro C et al. Profits and pandemics: prevention of harmful effects of tobacco, alcohol, and ultraprocessed food and drink industries. Lancet. 2013; 381(9867): 670-679.

3. Popkin BM, Hawkes C. Sweetening of the global diet, particularly beverages: patterns, trends, and policy responses. Lancet Diabetes Endocrinol. 2016; 4(2):174-186.

4. Freire WB, Waters WF, Rivas-Mariño G, Nguyen T, Rivas P. A qualitative study of consumer perceptions and use of traffic light food labelling in Ecuador. Public Health Nutr. 2017;20(5):805-813.

5. Keller KL, Staelin R. Effects of quality and quantity of information on decision effectiveness. J Consumer Res. 1987;14(2):200-13.

6. World Health Organization. Global Status Report of noncommunicable diseases 2014. Geneva: WHO; 2014.

7. Harvard School of Public Health. PURE study makes headlines, but the conclusions are misleading. Disponible en: https: / www. hsph.harvard.edu/nutritionsource/2017/09/08/pure-studymakes-headlines-but-the-conclusions-are-misleading/. Acceso el 18 de marzo de 2018

8. Fornasini M, Guevara D, Reyes S, López M, Cocha I, Morales M, Flores N, Aguirre S, Baldeon ME. Impact Of Milk Based Micronutrient Supplementation On Zinc, Iron, and Vitamin A Deficiencies In School Children In Quito, Ecuador. The Fasb J. 2016;30(1), Suppl. Disponible en: http://www.fasebj.org/doi/10.1096/ fasebj.30.1_supplement.669.12. Acceso el 18 de marzo de 2018

9. ILSI Nor-Andino. Disponible en: http:/ /ilsinorandino.org/organizacion/. Acceso el 22 de marzo de 2018

10. ILSI Nor-Andino. Asamblea Regional Ordinaria. 23 de marzo de 2017. Disponible en: http://ilsinorandino.org/wp-content/ uploads/sites/16/2018/03/Asamblea-Ordinaria-2017-website.pdf

11. Bes-Rastrollo M, Schulze MB, Ruiz-Canela M, Martinez-Gonzalez MA. Financial Conflicts of Interest and Reporting Bias Regarding the Association between Sugar-Sweetened Beverages and Weight Gain: A Systematic Review of Systematic Reviews. PLoS Med. 2013;10(12):e1001578 